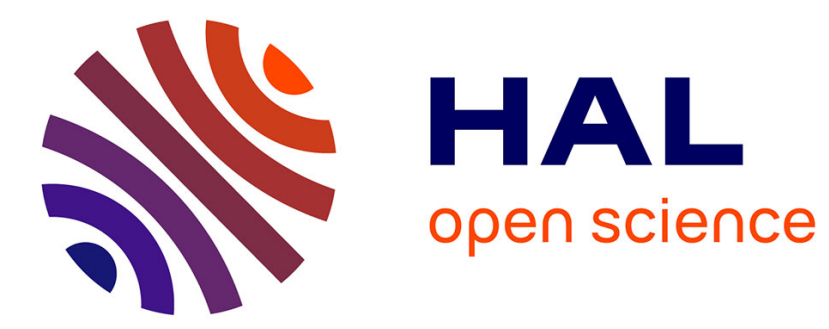

\title{
A Privacy Analysis of Google and Yandex Safe Browsing
}

Thomas Gerbet, Amrit Kumar, Cédric Lauradoux

\section{To cite this version:}

Thomas Gerbet, Amrit Kumar, Cédric Lauradoux. A Privacy Analysis of Google and Yandex Safe Browsing. [Research Report] RR-8686, INRIA. 2015. hal-01120186v4

\section{HAL Id: hal-01120186 \\ https://inria.hal.science/hal-01120186v4}

Submitted on 8 Sep 2015

HAL is a multi-disciplinary open access archive for the deposit and dissemination of scientific research documents, whether they are published or not. The documents may come from teaching and research institutions in France or abroad, or from public or private research centers.
L'archive ouverte pluridisciplinaire HAL, est destinée au dépôt et à la diffusion de documents scientifiques de niveau recherche, publiés ou non, émanant des établissements d'enseignement et de recherche français ou étrangers, des laboratoires publics ou privés. 
A Privacy Analysis of Google and Yandex Safe Browsing

Thomas Gerbet, Amrit Kumar, Cédric Lauradoux

RESEARCH

REPORT

$\mathrm{N}^{\circ} 8686$

February 2015

Project-Teams PRIVATICS 



\title{
Give

\section{A Privacy Analysis of Google and Yandex Safe Browsing}

\author{
Thomas Gerbet, Amrit Kumar, Cédric Lauradoux \\ Project-Teams PRIVATICS \\ Research Report $\mathrm{n}^{\circ} 8686$ - February 2015 - 24 pages
}

\begin{abstract}
Google and Yandex Safe Browsing are popular services included in many web browsers to prevent users from visiting phishing or malware website links. If Safe Browsing services protect their users from losing private information, they also require that their servers receive browsing information on the very same users. In this paper, we present an analysis of GoogLE and YANDEX Safe Browsing services from a privacy perspective. We quantify the privacy provided by these services by analyzing the possibility of re-identifying a URL visited by a client. We hence challenge GOOGLE's privacy policies where they claim that GOOGLE can not recover URLs visited by its users. Our analysis and experimental results show that Google and Yandex Safe Browsing can potentially be used as a tool to track specific classes of individuals. Additionally, our investigations on the data currently included in YANDEX Safe Browsing provides a concrete set of URLs/domains that can be re-identified without much effort.
\end{abstract}

Key-words: Malware, phishing, tracking

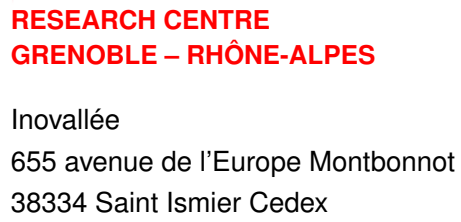




\section{A Privacy Analysis of Google and Yandex Safe Browsing}

Résumé : Les utilisateurs cliquent parfois par inadvertance sur des sites de phishing ou de malware et ce faisant ils sacrifient des informations secrètes et parfois même compromettent leur sécurité. Les créateurs de ces sites sont très motivés et sont capables de construire des URLs intelligemment scriptées. Compte tenu du nombre sans cesse croissant de tels URLs, de nouvelles stratégies ont été inventées pour les détecter et informer l'utilisateur final quand il est tenté d'accéder à un tel lien. Au premier rang de ces techniques se trouve les services de Safe Browsing, qui interrogent une liste noire de sites dangereux et qui avertissent les utilisateurs leur permettant de se protéger.

Dans cet article, nous montrons comment les services Safe Browsing endommagent la vie privée de leurs utilisateurs. Nous étudions la vie privée de systèmes GoOGLE et YANDEx pour comprendre quelles informations ils peuvent recueillir. Nous avertissons leurs utilisateurs que ces les services peuvent être facilement utilisés pour la surveillance de suivre des classes spécifiques d'individus ou de censurer certains sites. GOOGLE et YANDEX ne sont pas actuellement responsables des entrées inclus dans leur liste noire. Jusqu'à une avancée majeure dans le domaine de la recherche d'informations privées, les services de Safe Browsing ne peuvent pas être respectueux de la vie privé. Nous fournissons des preuves que YANDEX a toutes les sondes en place pour suivre les utilisateurs de sites Web ciblés.

Mots-clés : Malware, phishing, surveillance 


\section{Introduction}

In 2008, Google started a service called Google Safe Browsing (GSB) Incc to warn and dissuade an end user from visiting phishing and malware web pages. With similar goals, YANDEX followed up with an identical service named YANDEX Safe Browsing (YSB). As of today, all major web browsers including Firefox, Internet Explorer, Safari, Opera and Yandex.Browser feature one of these Safe Browsing (SB) services. The integration of SB services in the browsers has naturally generated an extremely large user base. GSB alone accounts to a billion users until date [Inc14].

The first version of GSB aka the Lookup API, raised many privacy concerns: URLs were sent in clear to the GOOGLE servers. GOOGLE could potentially capture the browsing history of GSB users. GOOGLE later came up with a new version of GSB that was conceived as a more privacy-friendly service. The same architecture has been adopted by YANDEX for YSB. Essentially, in this new version, a GSB client computes a cryptographic digest of a given URL and checks if its 32-bit prefix matches a local database of malicious prefixes provided by GooGLE. A database miss ensures that the URL is safe. While, a hit, requires the client to query the server by sending the prefix to eliminate false positives. From the server's reply, the client can eventually determine whether the URL is malicious or not. Google Chrome Privacy Notice Goo14 includes a section on GSB. It states that:

"Google cannot determine the real URL from this information (read prefix)."

Google reiterates this statement in a document concerning the GSB usage in Mozilla Firefox Goo12. These guarantees have allowed GSB to be massively used by the end users and even by other web service providers.

Apart from these statements from Google, there is no other privacy analysis of GSB. The goal of this paper is to provide an independent privacy analysis of GSB and its sibling YSB. To this end, we first provide a comprehensive description of GOOGLE and YANDEX Safe Browsing in Section 2 and Section 3 respectively. In Section 4, we present a threat model for re-identification and tracking. The SB services employ an anonymization technique that is a combination of hashing and truncation. Hashing is used to create pseudonyms for URLs. Generating pseudonyms (digests) of the URLs however does not suffice to anonymize the data, and hence truncation is applied to create collisions. Truncation of these pseudonyms ensure that several URLs share the same reduced pseudonym (prefix). We quantify the privacy provided by this solution using a balls-into-bins argument and $k$-anonymity Swe02 when a single prefix is sent to the servers for a URL (Section 5).

It appears that multiple prefixes can be sent to the servers for a given URL. Indeed, a client does not simply compute the digest of a URL, but instead, computes the digests of all its decompositions. Distinct URLs can share common prefixes in their decompositions. We characterize the different cases for such multiple collisions in Section 6 and collect statistics on URLs using data from Common Crawl. Our experimental analysis estimates the rate of such collisions and shows that hashing and truncation fails to prevent re-identification when a user visits small-sized domains or certain URLs of larger domains. We further materialize this in the form of an algorithm that GoOGLE and YANDEX could potentially employ to track users.

We conclude this work by providing an analysis of the databases of GoogLE and YANDEX (Section 7). By crawling their databases, we detect a number of "suspicious" prefixes that we call orphans. Orphans trigger communication with the servers, but no full digest corresponds to them. We also observe several URLs which have multiples prefixes included in the blacklists. These provide concrete examples of URLs and domains that can be easily tracked by GoOGLE and YANDEX. 


\section{Google Safe Browsing}

GSB aims to provide a comprehensive and timely detection of new threats on the Internet.

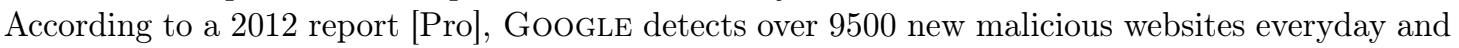
provides warnings for about 300 thousand downloads per day. We highlight that the GSB service is not restricted to search, but it also extends to filter malicious adds. It has further opened paths for new services such as instantaneous phishing and download protection, i.e., protection against malicious drive-by-downloads, Chrome extension for malware scanning and Android application protection.

In this section, we first describe our motivation to study and analyze GSB and then we present a comprehensive description of the GSB architecture.

\subsection{Motivations}

Our choice to work on GSB is motivated by two factors. First, being that it is one of the only two SB services designed to be privacy friendly. The other service being YSB, which replicates the GSB architecture. The second is its high impact on users and other web services.

Indeed, many software vendors have proposed solutions for SB. Microsoft promotes SmartScreen URL Filter Cor13 and ships it with its products including Internet Explorer, Outlook.com, Outlook, Exchange, Windows Live Mail and Entourage. Web of Trust (WOT) [WSa, and Norton Safe Web (NSW) Sym, developed by Symantec are tools operating as a web browser plugin. Finally, McAfee SiteAdvisor $\mathrm{McA}$ is a product very similar to NSW.

In a general manner, all SB services filter malicious links by relying on a dynamic blacklist of malicious URLs. However, all the afore-listed SB providers except GoOGLE and YANDEX provide an SB service that is privacy-unfriendly by design: the URL or a part of it is sent in clear to the servers during the lookup in the blacklists. Furthermore, most of these SB providers agree in their privacy policies that they receive the URLs, but that they are not used to identify, contact, or target adds to users. This is the only guarantee that the users have. To our knowledge, Google and therefore YANDEX Safe Browsing are the only SB services with built-in privacy features.

Our second motivation to work on GSB stems from its popularity: it has been included in all major browsers namely Chrome, Chromium, Firefox, Safari and Opera. According to STATCOUNTER (statcounter.com), these represent $65 \%$ of all the browsers in use. Several other independent web services such as TwitTER and BITLY also employ GSB to prevent users from disseminating malicious URLs. FACEBOOK has developed a phishing and malware filter called Link Shim Inca that extensively relies on GSB and WOT. Fig. 1 schematically presents the major clients of GSB and YSB.

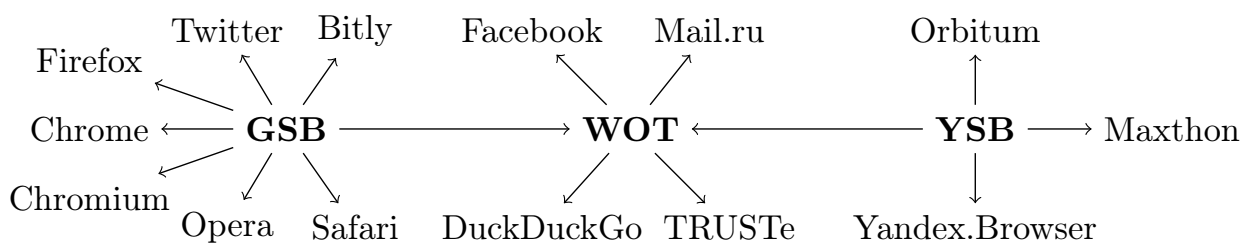

Figure 1: The Safe Browsing ecosystem. 


\subsection{Description}

The essential goal of GSB is to warn and dissuade an end user from visiting malicious URLs. The service is implemented at the application layer (HTTP level) of the standard Internet stack. Consequently, whenever a client (typically a browser) attempts to visit a malicious URL, the client can display an interstitial warning page before the suspicious web page is actually requested.

In Fig. 2, we present a simplified architecture of the GSB service. GooGLE crawlers harvest malicious URLs from the web and then transmit them to the GSB servers. Clients may then consult the server to check if a link is malicious.

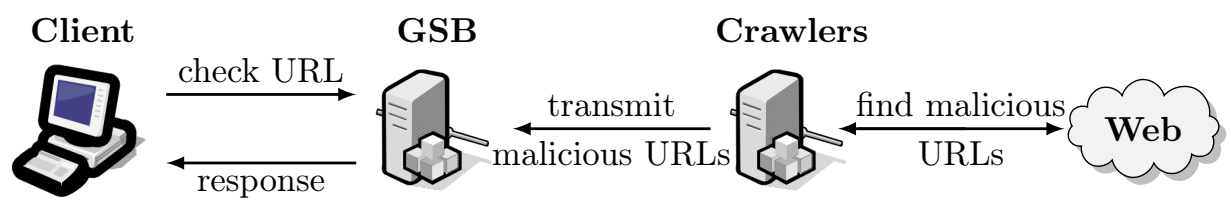

Figure 2: High level overview of Google Safe Browsing.

GSB classifies malicious URLs into two main categories: malware and phishing. The blacklists and their number of entries are given in Table 1. They contain SHA-256 digests of malicious URLs. The lists can either be downloaded partially to only update a local copy or can be obtained in its entirety.

Table 1: Lists provided by Google Safe Browsing API. Information could not be obtained for cells marked with $(*)$.

\begin{tabular}{l||l|r} 
List name & Description & \#prefixes \\
\hline \hline goog-malware-shavar & malware & 317,807 \\
\hline goog-regtest-shavar & test file & 29,667 \\
\hline goog-unwanted-shavar & unwanted softw. & $*$ \\
\hline goog-whitedomain-shavar & unused & 1 \\
\hline googpub-phish-shavar & phishing & 312,621 \\
\hline
\end{tabular}

GSB was first conceived in the form of a Lookup API. Using this API, a client could send the URL to check using HTTP GET or POST requests and the server performed a look up in the malicious lists. However, the API was soon declared deprecated for privacy and efficiency considerations. This was mainly because URLs were sent in clear to the servers and each request implied latency due to the network round-trip. To address these issues, Google currently offers another API: Google Safe Browsing API, described below.

\subsubsection{Safe Browsing API v3}

The Google Safe Browsing API v3 is now the reference API to use GSB. It has been positively received by the community as a major improvement for privacy.

In contrast to the Lookup API, the client now does not handle a URL directly. Instead, the URL is canonicalized following the URI specifications BLFM05 and then hashed with SHA-256 Nat12. The digest is then checked against a locally stored database which contains 32-bit prefixes of malicious URL digests. If the prefix is not found to be present in the local database, then the URL can be considered safe. However, if there is a match, the queried URL may not necessarily be malicious: it can be a false positive. Consequently, the client must query the GSB server by sending the prefix. The server in response sends all the full digests 
corresponding to the received prefix. Finally, if the full digest of the client's prefix is not present in the list returned by the server, the URL can be considered safe. Fig. 3 summarizes a request through the GSB API.

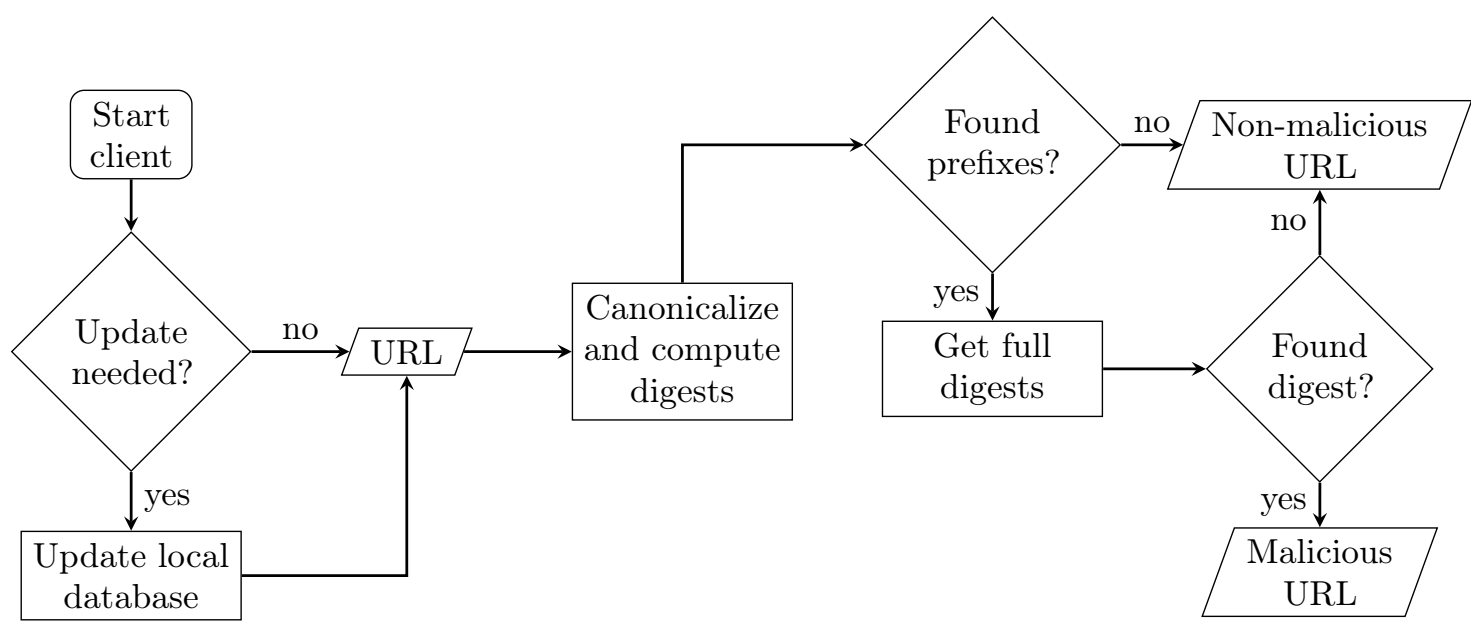

Figure 3: Google Safe Browsing: Client's behavior flow chart.

We note that the lookup may require a client to test several URLs for a target URL. This is because the complete URL might not have been included in the blacklists. Let us consider the most generic HTTP URL of the form http://usr:pwd@a.b.c:port/1/2.ext?param=1\#frags (see [BLMM94]), where usr is a username and pwd is the corresponding password, a.b.c is a fully-qualified domain name, port is a TCP or UDP port number, 1/2.ext is the URL path, ?param=1 is the query and \#frags identifies the particular place within a remote resource. Then, in order to check whether the URL is malicious, the client first performs a canonicalization of the URL and then performs a lookup for the following 8 decompositions in the given order:
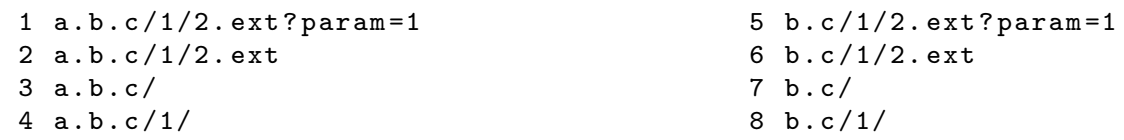

If any of the above URLs creates a hit in the local database, then the initial link is considered as suspicious and the prefix can be forwarded to the GoogLE server for a confirmation. If there are more than 1 hits, then all the corresponding prefixes are sent. After receiving the list of full digests corresponding to the suspected prefixes fragments, they are locally stored until an update discards them. Storing the full digests prevents the network from slowing down due to frequent requests. To maintain the quality of service and limiting the amount of resources needed to run the API, Google has defined for each type of requests (malware or phishing) the frequency of queries that clients must restrain to.

\subsubsection{Local Data Structures}

The choice of the data structure to store the prefixes on the client's side is constrained by two factors: fast query time and low memory footprint. Google has deployed two different data structures until now: Bloom filters [Blo70] and Delta-coded tables $\left[\mathrm{MKD}^{+} 02\right]$.

In an early version of Chromium (discontinued since September 2012), a Bloom filter was used to store the prefixes on the client's side. This solution was abandoned to be replaced by 
delta-coded tables. Unlike the classical Bloom filters, this data structure is dynamic, does not have any "intrinsic" false positive probability and yet incurs a lower memory footprint. However, its query time is slower than that of Bloom filters. Even though delta-coded tables do not entail any intrinsic false positive probability, their use to store 32-bit prefixes still generates false positives. False positives arise since several URLs may share the same 32-bit prefix.

We have implemented both the data structures to understand why Bloom filters were abandoned and why Google has chosen 32-bit prefixes. The results are shown in Table 2 , If 32-bit prefixes are stored, the raw data requires $2.5 \mathrm{MB}$ of space. Storing these prefixes using a delta-coded table only requires $1.3 \mathrm{MB}$ of memory, hence GoOGLE achieves a compression ratio of 1.9. Bloom filters are immune to the change in the prefix size, and thus require a larger but constant space of $3 \mathrm{MB}$. However, starting from 64-bit prefixes, Bloom filters outperform delta-coded tables. The low memory footprint of Bloom filters comes at the cost of being a static data structure, which makes them unsuitable for the highly dynamic blacklists provided by Google. This justifies Google's choice of delta-coded tables over Bloom filters and the choice of 32-bit prefixes.

Table 2: Client's cache size for different prefix sizes.

\begin{tabular}{|c|c|c|c|}
\cline { 3 - 4 } \multicolumn{2}{c|}{} & \multicolumn{2}{c|}{ Data structure (MB) } \\
\hline Prefix (bits) & Raw data (MB) & Delta-coded & Bloom \\
\hline 32 & 2.5 & 1.3 & \\
64 & 5.1 & 3.9 & \\
80 & 6.4 & 5.1 & 3 \\
128 & 10.2 & 8.9 & \\
256 & 20.3 & 19.1 & \\
\hline
\end{tabular}

\subsubsection{Safe Browsing Cookie}

GSB has often been criticized ever since web browsers have started to use them. This is essentially because when implemented inside web browsers, each request to the API also sends a cookie which identifies a client. The cookie sent by the browsers is the same as the one used by other services provided by GoOGLE especially the social features such as the +1 button. To these criticisms, GOOGLE responded that the cookies were not used to track users but only to monitor the performance of the service on the server-side and to catch bugs (see [goo]) Since they are needed by Google to operate the service, the browsers can not disable it. However, Chromium and Firefox have isolated the SB cookie from the others with the purpose of achieving maximum privacy (see $\mathrm{moz})$.

\section{Yandex Safe Browsing}

The YANDEx Safe Browsing (YSB) service comes in the form of an API [Yan] and also as a security feature in its browser called Yandex.Browser. The YAnDEx Safe Browsing API is compatible with C\#, Python and PHP and is a verbatim copy of the GSB API with the only difference that in addition to the phishing and the malware lists provided by GoogLE, the YSB API also includes 17 other blacklists. Each of these lists contains malicious or unsafe links of a given category.

Table 3 provides the name and description of the blacklists with the number of prefixes present in each. We highlight that the malware blacklists goog-malware-shavar and ydx-malware-shavar are identical. The same holds for the malware prefix blacklists meant for mobile devices: 
goog-mobile-only-malware-shavar and ydx-mobile-only-malware-shavar, and for phishing: goog-phish-shavar and ydx-phish-shavar. By comparing the goog-malware-shavar lists of GoOgLE and YANDEX, we observed that there are only 36547 prefixes in common. Similarly, the phishing lists googpub-phish-shavar and goog-phish-shavar of GOOGLE and YANDEX respectively have only 195 prefixes in common. These anomalies exist because the lists might not be up-to-date on the YANDEX server.

Table 3: Yandex Blacklists. Information could not be obtained for cells marked with $\left(^{*}\right)$.

\begin{tabular}{l||l|r}
\multicolumn{1}{l||}{ List name } & Description & \#prefixes \\
\hline \hline goog-malware-shavar & malware & 283,211 \\
\hline goog-mobile-only-malware-shavar & mobile malware & 2,107 \\
\hline goog-phish-shavar & phishing & 31,593 \\
\hline ydx-adult-shavar & adult website & 434 \\
\hline ydx-adult-testing-shavar & test file & 535 \\
\hline ydx-imgs-shavar & malicious image & 0 \\
\hline ydx-malware-shavar & malware & 283,211 \\
\hline ydx-mitb-masks-shavar & man-in-the-browser & 87 \\
\hline ydx-mobile-only-malware-shavar & malware & 2,107 \\
\hline ydx-phish-shavar & phishing & 31,593 \\
\hline ydx-porno-hosts-top-shavar & pornography & 99,990 \\
\hline ydx-sms-fraud-shavar & sms fraud & 10,609 \\
\hline ydx-test-shavar & test file & 0 \\
\hline ydx-yellow-shavar & shocking content & 209 \\
\hline ydx-yellow-testing-shavar & test file & 370 \\
\hline ydx-badcrxids-digestvar & .crx file ids & $*$ \\
\hline ydx-badbin-digestvar & malicious binary & $*$ \\
\hline ydx-mitb-uids & man-in-the-browser & $*$ \\
\hline ydx-badcrxids-testing-digestvar & test file & $*$ android app UID \\
\hline
\end{tabular}

\section{Threat Model}

The clients using SB tools and especially web browsers are exposed to several privacy threats. An honest-but-curious SB provider can reconstruct completely or partly the browsing history of a client from the data sent to the servers. As previously mentioned, this is the case for most SB tools. This is also why the Lookup API of GSB was rejected by the community.

Another threat posed by SB services consists in revealing if a client has visited some selected webpages and not the full browsing history. By associating traits to pages, the ultimate goal of the provider is to detect users' behavior such as political opinions, sexual orientation or terrorism.

We note that the capabilities of SB providers are often immense and that we cannot restrict our adversary to the honest-but-curious model. To reach their goal, we assume that the provider is willing to do anything including tampering with the database of the service. In GSB and YSB, the providers may on their own initiative include values in the prefix database of the client to track them or they can be constrained by a third party (governmental agencies) to do so. Since, GOOGLE and YANDEX have web indexing capabilities, we safely assume that they maintain the database of all webpages and URLs on the web. We further assume that the SB servers may aggregate requests for full hashes and exploit the temporal correlation between the queries.

In the following, we analyze GSB and YSB for the afore-mentioned threats. To this end, we consider two cases which differ from the number of prefixes requested by the client to the server. 


\section{$5 \quad$ Single Prefix Match}

A simple way to understand GSB and YSB consists in considering them as a probabilistic test run by the client to filter malicious URLs. As a probabilistic solution, this entails true positives, false positives, etc. Hence, whenever the test executed by the browser is positive, Google or YANDEX servers are contacted to remove any ambiguity. While a negative test leaks no information about the URL to GOOGLE and YANDEx, a positive test sends prefix(es) of certain decompositions of the target URL. The exact number of prefixes sent depends on the number of hits in the local database.

In this section, we analyze the privacy provided GSB and YSB when their servers receive one single prefix corresponding to a URL. Studying this case allows us to know if a user's browsing history can be fully/partly constructed by GoOGLE and YANDEX.

\subsection{Privacy Metric}

In order to exemplify the evoked privacy concern and define a privacy metric, let us consider the PETS CFP URL: https://petsymposium.org/2016/cfp.php. Its decompositions are shown in Table 4. We suppose that the first decomposition creates a hit in the local database, i.e., 0xe70ee6d 1 is present in one of the blacklists.

Table 4: Decompositions of PETS CFP URL and the prefixes.

\begin{tabular}{|l|l|}
\hline URL & 32-bit prefix \\
\hline \hline petsymposium.org/2016/cfp.php & 0xe70ee6d1 \\
\hline petsymposium.org/2016/ & 0x1d13ba6a \\
\hline petsymposium.org/ & 0x33a02ef5 \\
\hline
\end{tabular}

Let us consider a situation where a client visits a web page that creates a hit on the prefix 0xe70ee6d1. Hence, it sends the prefix to the server. In order to determine if this prefix is enough for GOOGLE or YANDEX to re-identify the corresponding URL, we consider the following privacy metric. The metric is defined as the maximum number of URLs which share a given prefix. This measures the worst-case uncertainty for SB providers in re-identifying the URL from a prefix. Higher is the value of this maximum, better is the privacy achieved. This privacy metric yields a simple yet reliable method to analyze and quantify the information leakage through prefixes. Furthermore, the metric may be viewed as a $k$-anonymity argument Swe02 to support the fact that the URLs are anonymized through hashing-and-truncation.

\subsection{Analysis}

In this section, we compute the afore-defined privacy metric when the server receives one prefix corresponding to a URL. It is worth noticing that one may argue that there are infinite number of pre-images for a 32-bit prefix, hence the privacy metric that estimates the uncertainty in re-identification should be infinitely large. However, the crucial point here is that the total number of URLs on the web is finite and hence the privacy metric can only be finitely small. In the following, we use a result from Raab and Steger [RS98] on balls-into-bins to compute the maximum number of URLs that can share the same $\ell$-bit prefix. In our context, prefixes represent the bins and the URLs are considered as the balls.

Theorem 1 (Raab and Steger [RS98|) Let $M$ be the random variable that counts the maximum number of balls into any bin. If we throw $m$ balls independently and uniformly at random 
into $n=2^{\ell}$ bins, then $\operatorname{Pr}\left[M>k_{\alpha}\right]=o(1)$ if $\alpha>1$ and $\operatorname{Pr}\left[M>k_{\alpha}\right]=1-o(1)$ if $0<\alpha<1$, where:

$$
k_{\alpha}= \begin{cases}\frac{\log n}{\log \frac{n \log n}{m}}\left(1+\alpha \frac{\log ^{(2)}\left(\frac{n \log n}{m}\right)}{\log \frac{n \log n}{m}}\right), & \text { if } \frac{n}{\operatorname{polylog}(n)} \leq m \ll n \log n, \\ \left(d_{c}-1-\alpha\right) \log n, & \text { if } m=c \cdot n \log n, \\ \frac{m}{n}+\alpha \sqrt{2 \frac{m}{n} \log n}, & \text { if } n \log n \ll m \leq n \cdot \operatorname{polyl} \log (n), \\ \frac{m}{n}+\sqrt{\frac{2 m \log n}{n}\left(1-\frac{1}{\alpha} \frac{\log \left(^{(2)} n\right.}{2 \log n}\right),} & \text { if } m \gg n \cdot(\log n)^{3} .\end{cases}
$$

The equation for computing $d_{c}$ can be found in RS98.

If $n=2^{\ell}$ is the number of possible prefixes and $m$ is the number of unique URLs, then $M$ is the maximum number of URLs matching a given prefix. This measures the worst-case uncertainty for GoOGLE or YANDEX in re-identifying a URL from a received prefix. A more correct argument from the client's point of view would be to consider the bin having the least number of balls. Higher is the overall minimum, lower is probability that a re-identification succeeds. According to the result of Ercal-Ozkaya [EO08, for a constant $c>1$, and $m \geq c n \log n$, the minimum number of balls in any bin is $\Theta\left(\frac{m}{n}\right)$. We however take the attacker's perspective: for Google and YANDEX, $M$ is the worst case uncertainty for URL re-identification.

In 2008, Google Incb claimed to know 1 trillion unique URLs. Since then, Google officials have reported 30 trillion URLs in 2012 and 60 trillion in 2013. These data are summarized in the first two rows of Table 5. The table also presents the number of domain names provided by VERISIGN in its reports [ncd].

Using Theorem 1 and the provided Internet data, we compute the values $M$ for unique URLs and domain names for different prefix sizes over the last few years. Results are provided in Table 5. When GSB was started in 2008, at most 443 URLs matched a given 32-bit prefix. It has increased over the years to reach 14757 in 2013. In the worst case, it is hard for GoogLE and YANDEX to re-identify a URL from a single 32-bit prefix. The case of domain names is slightly different because the space of domain names is much smaller and its dynamic is far slower than the one of URLs. In the worst case, two domain names will collide to the same prefix. Domain names can hence be re-identified with high certainty. However, the server does not know if the received prefix corresponds to a domain name or to a URL.

Table 5: $M$ for URLs and domains with different prefix sizes $\ell$.

\begin{tabular}{|r|r|r|r||r|r|r|}
\cline { 2 - 8 } \multicolumn{1}{c|}{} & \multicolumn{3}{c||}{ URLs $\left(10^{12}\right)$} & \multicolumn{3}{c|}{ domains $\left(10^{6}\right)$} \\
\hline Year & 2008 & 2012 & 2013 & 2008 & 2012 & 2013 \\
\hline Number & 1 & 30 & 60 & 177 & 252 & 271 \\
\hline \hline$\ell$ (bits) & \multicolumn{3}{|c||}{$M$} & \multicolumn{3}{|c|}{$M$} \\
\hline \hline 16 & $2^{28}$ & $2^{28}$ & $2^{29}$ & 3101 & 4196 & 4498 \\
\hline 32 & 443 & 7541 & 14757 & 2 & 3 & 3 \\
\hline 64 & 2 & 2 & 2 & 1 & 1 & 1 \\
\hline 96 & 1 & 1 & 1 & 1 & 1 & 1 \\
\hline
\end{tabular}

Hence, a single prefix per URL does not allow the SB server to reconstruct the browsing history of the client. So far, the solution seems to be privacy preserving as long as the client only reveals a single prefix. 


\section{Multiple Prefix Match}

In this section, we analyze the case when the backend SB servers receive multiple prefixes. SB servers may receive multiple prefixes for three reasons. First, it could be the result of accidental hits in the local database. This may happen when several decompositions of a non-malicious URL create hits in the database. Second, the SB providers may aggregate prefixes received over time. This could mainly be done to exploit any temporal correlation between the queries. Third and most importantly, in certain cases an SB provider may be forced to include several prefixes for a target URL in the local database. This might be necessary when a domain has a subset of sub-domains and URL paths which host several malicious URLs. Then the sub-domains and the paths can be blacklisted instead of including each malicious URL in the database. This approach saves memory footprint on the client's side. Consequently, whenever a client visits any of the malicious URLs, multiple prefixes are sent to the servers. We note that one could possibly include only the prefix of the domain to blacklist all its malicious sub-domains and paths. However, this approach blacklists all non-malicious URLs on the domain. Multiple prefixes are hence indispensable to prevent certain URLs from being flagged as malicious.

Multiple prefix match for a URL forces the client to send more information to the servers than in the case of a single prefix match. Clearly, the amount of information on the URL obtained by the server is proportional to the actual number of prefixes received or aggregated. In the sequel, we analyze whether multiple prefixes may allow GSB and YSB to re-identify the URL visited by a client.

\subsection{URL Re-identification}

In order to present a comprehensible analysis of re-identification, we henceforth consider the simplified case of 2 prefixes. The analysis for the case when the server receives more than 2 prefixes per URL follows in a straightforward manner.

As in the single prefix case, more than two distinct URLs may yield the same two prefixes. The larger is the number of such URLs, the more difficult is the re-identification. These URLs exist due to three possible types of collisions on 32-bit prefixes. In the first type (Type I), several distinct yet related URLs share common decompositions and these decompositions yield the shared prefixes. We note that 2 distinct URLs are related if they have common sub-domains. The second type of collisions (Type II) is due to distinct yet related URLs that share one decomposition and hence one common prefix, while the other common prefix is due to the collision on truncated digests. Finally, the last type of collisions (Type III) appears when completely unrelated URLs generate the same prefixes. The latter may occur again due to collisions on the truncated digests. In the following, by a Type I URL, we mean a URL that generates a Type I collision with a given URL. We similarly define Type II and Type III URLs for a given URL.

In order to illustrate the different possible collisions, we present a set of examples in Table6. We assume that the client visits the target URL a.b.c and hence the server receives the corresponding two prefixes, say $A$ and $B$. The server using these prefixes must determine the exact URL visited by the client. The next three URLs exemplify the different types of collisions.

Clearly, the following inequalities among the probabilities hold:

$$
\mathbb{P}[\text { Type } \mathrm{I}]>\mathbb{P}[\text { Type } \mathrm{II}]>\mathbb{P}[\text { Type III }] \text {, }
$$

where $\mathbb{P}[X]$ denotes the probability of an event $X$. Under the uniformity assumption of hash functions, a Type III collision is highly unlikely, with a probability of $\frac{1}{2^{64}}$. We note that for Type I and Type II collisions to occur, the URLs must share at least one common decomposition. The probability of these collisions hence depends on the number of decompositions of URLs hosted on 
Table 6: An illustrative example with different possible collisions.

\begin{tabular}{|c|l|r|r|c|}
\cline { 3 - 5 } \multicolumn{1}{c|}{} & URL & Decomp. & Prefixes \\
\cline { 2 - 5 } \multicolumn{1}{c|}{} & \multirow{2}{*}{ Target URL } & \multirow{2}{*}{ a.b.c } & a.b.c/ & $A$ \\
& & b.c/ & $B$ \\
\hline \multirow{4}{*}{ Coll. Type } & \multirow{3}{*}{ Type I } & g.a.b.c & g.a.b.c/ & $C$ \\
& & & a.b.c/ & $A$ \\
& \multirow{3}{*}{ Type II } & \multirow{2}{*}{ g.b.c/ } & g.b.c/ & $B$ \\
\cline { 3 - 5 } & & & b.c/ & $B$ \\
\cline { 2 - 5 } & \multirow{2}{*}{ Type III } & \multirow{2}{*}{ d.e.f } & d.e.f/ & $A$ \\
& & & e.f/ & $B$ \\
\hline
\end{tabular}

the domain. In general, smaller is the number of decompositions per URL, lower is the probability that Type I and Type II URLs exist. Moreover, a Type II URL exists only if the number of decompositions on a domain is larger than $2^{32}$. In the next section, we show that no Type II URL exists by empirically estimating the distribution of decompositions over domains.

As a result, the ambiguity in the re-identification can only arise due to Type I collisions. Moreover, a target URL with few decompositions has a very low probability to yield Type I collisions and hence it can be re-identified with high probability. In case of URLs with large number of decompositions, the server would require more than 2 prefixes per URL to remove the ambiguity. Nevertheless, the SB provider can still determine the common sub-domain visited by the client using only 2 prefixes. This information may often suffice to identify suspicious behavior when the domain in question pertains to specific traits such as pedophilia or terrorism. It is pertinent to highlight that the SB service provided by WOT collects the domains visited by its clients $[\mathrm{WSb}]$. Hence, in the scenario when GSB and YSB servers receive multiple prefixes for a URL, the privacy achieved is the same as that ensured by services such as WOT.

Now, let us further analyze the problem of re-identifying URLs for which Type I collisions occur. To this end, we consider an illustrative example of a domain b.c that hosts a URL a.b.c/1 and its decompositions (see Table 7). We assume that these are the only URLs on the domain. The URL generates four decompositions. Two of these decompositions include the domain name 'a' as a sub-domain while the remaining two do not. These decompositions yield prefixes say $A$, $B, C$ and $D$ respectively. We note that the considered URL is only a slightly simplified form of the most general URL, where the query part of the URL has been removed BLMM94. Therefore, it largely represents all the canonicalized URLs.

Table 7: A Sample URL on the host b.c with its 4 decompositions.

\begin{tabular}{c|l|c} 
URL & Decompositions & Prefix \\
\hline \hline \multirow{4}{*}{ a.b.c/1 1} & a.b.c/1 & $A$ \\
& a.b.c/ & $B$ \\
\cline { 2 - 3 } & b.c $/ 1$ & $C$ \\
& b.c/ & $D$ \\
\hline
\end{tabular}

We analyze the following three cases depending on the prefixes sent by the client to the SB server:

Case 1. $(A, B)$ generate hits: In this case, both the corresponding decompositions contain the domain name ' $a$ '. Hence, if the server receives these prefixes, it can be sure that the client 
has visited the URL that corresponds to the first prefix $A$, i.e, a.b.c/1. The probability that the re-identification fails is $\mathbb{P}[$ Type III $]=\frac{1}{2^{64}}$.

Case 2. $(C, D)$ generate hits: These prefixes correspond to those URLs that do not contain the domain name ' $a$ '. In this case, the possible URLs that the client could have visited are: a.b.c/1, a.b.c/ or b.c/1. These correspond to the URLs yielding the prefixes $A$, $B$ and $C$ respectively. Hence, in order to remove the ambiguity and re-identify the exact URL visited by the client, the SB provider would include additional prefixes in the local database. If it includes the prefix $A$, in addition to $C$ and $D$, then it can learn whether the client visited the URL a.b.c/1 or b.c/1. More precisely, if the client visits a.b.c/1 then prefixes $A, C$ and $D$ will be sent to the server, while if the client visits b.c/1, then only $C$ and $D$ will be sent. Similarly, in order to distinguish whether the client visits a.b.c/ or b.c/, the SB provider would additionally include the prefix $B$.

Case 3. $\{A, B\} \times\{C, D\}$ generate hits: If the prefix $A$ creates a hit, then the URL visited by the client is certainly a.b.c/1, while if the prefix $B$ creates a hit then the client has either visited a.b.c/1 or a.b.c/. As in the previous case, the re-identification requires the SB provider to include an additional prefix $A$ in the prefix database.

As a general rule, all the decompositions that appear before the first prefix are possible candidates for re-identification. Consequently, lower-level domain names and URL paths can be re-identified with a higher certainty than the ones at a higher level. To this end, we consider the case of leaf URLs on a domain. We call a URL on a given domain a leaf, if it does not belong to the set of decompositions of any URL hosted on the domain. A leaf URL can also be identified as a leaf node in the domain hierarchy (see Figure 4). Type I collisions for these URLs can be easily eliminated during re-identification with the help of only two prefixes. The first prefix corresponds to that of the URL itself, while the other one may arbitrarily correspond to any of its decompositions. In the example of Table 7] the URL a.b.c/1 is a leaf URL on the domain b.c, and hence it can be re-identified using prefixes $(A, B)$.

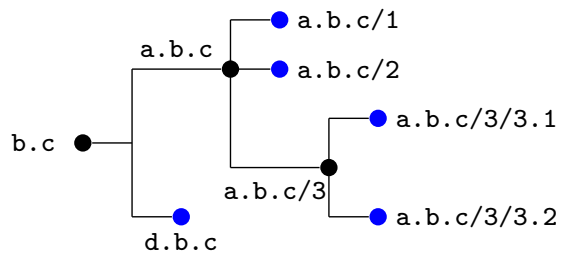

Figure 4: A sample domain hierarchy. Leaf URLs are shown in blue.

Clearly, only non-leaf URLs contribute to Type I collisions. Hence, in order to re-identify non-leaf nodes, one must include more than 2 prefixes per node. Our observations further raise the question of the distribution of decompositions over domains that we explore in the following section.

\subsection{Statistics on Decompositions}

Results from the previous sections show that a URL is largely re-identifiable if more than one of the prefixes corresponding to its decompositions are included in the local database. It is worth noticing that popular domains often host more URLs than the non-popular ones. This 


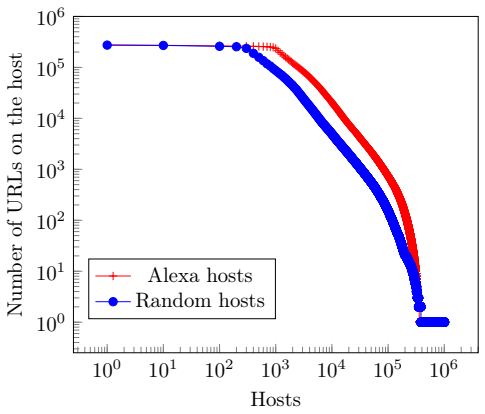

(a)

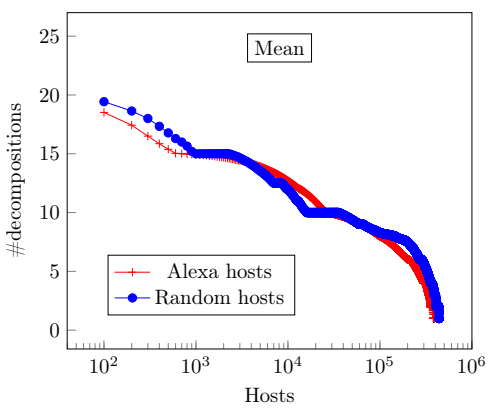

(d)

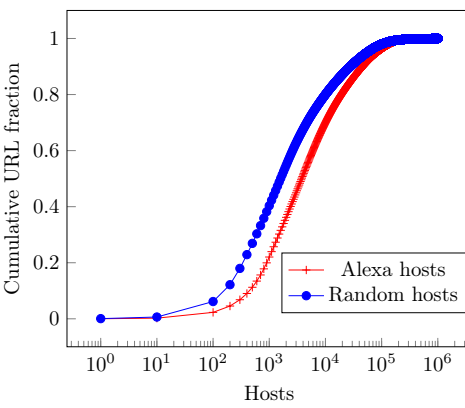

(b)

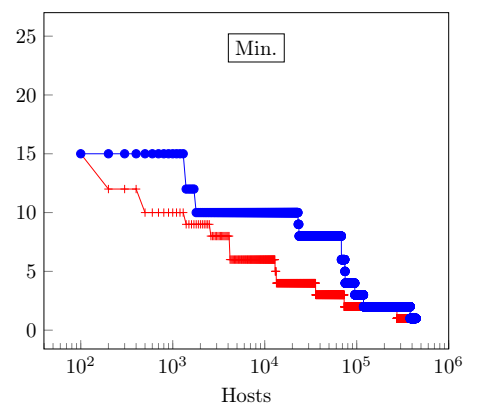

(e)

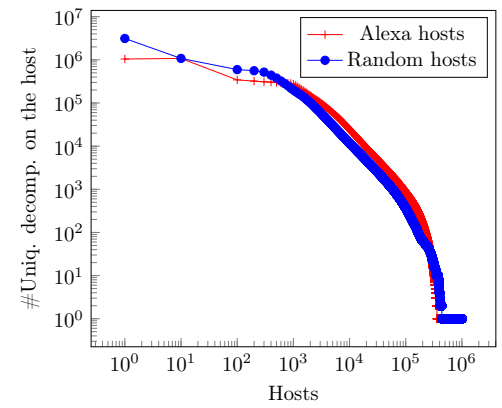

(c)

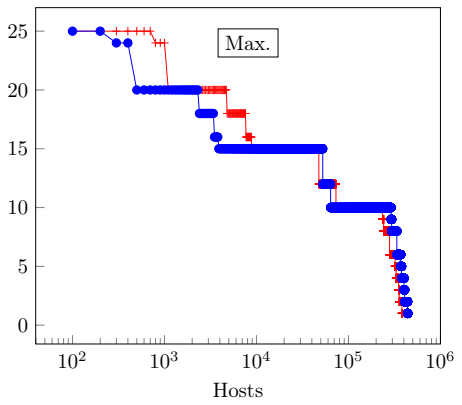

(f)

Figure 5: Distribution of URLs and decompositions on hosts from the two datasets. Figure (a) presents the distribution of URLs over hosts, while (b) presents its cumulative distribution. (c) shows the distribution of decompositions over hosts. (d), (e) and (f) present the mean, minimum and maximum number of URL decompositions on the hosts.

generally implies that the number of unique URL decompositions and eventual collisions on popular domains are larger than those on non-popular ones.

We experimentally estimate the distribution of URLs, decompositions and collisions over domains with the help of the web corpus provided by Common Crawl com15]. The Common Crawl is an open repository of web crawl data collected over the last 7 years. It contains raw web page data, metadata and text extractions. For our experiments, we have used the most recent corpus of April 2015. This crawl archive is over $168 \mathrm{~TB}$ in size and holds more than 2.11 billion web pages.

We consider two datasets in our experiments. Our first dataset contains web pages on the 1 million most popular domains of Alexa ale15]. We also collected 1 million random domains from the common crawl and then recovered web pages hosted on these domains. This forms our second dataset. The number of URLs and the total number of decompositions provided by these datasets is given in Table 8. Our dataset of URLs on popular domains contains around 1.2 billion URLs, while the dataset on random domains includes over 427 million URLs. The URLs in the Alexa dataset yield around 1.4 billion unique decompositions in total, while the random dataset generates around 1 billion decompositions.

In the following, we present our findings on these two datasets. Figure $5 \mathrm{a}$ presents the number of URLs hosted on the domains belonging to these two datasets. Clearly, the Alexa domains host larger number of URLs than the random domains. The most number of URLs hosted by a 
Table 8: Our datasets.

\begin{tabular}{l|r|r|r}
\hline Dataset & \#Domains & \#URLs & \#Decompositions \\
\hline \hline Alexa & $1,000,000$ & $1,164,781,417$ & $1,398,540,752$ \\
\hline Random & $1,000,000$ & $427,675,207$ & $1,020,641,929$ \\
\hline
\end{tabular}

domain from either of the datasets is around $2.7 \times 10^{5}$. This peak is due to the fact that crawlers do not systematically collect more pages per site than this bound due to limitations imposed by the server. We also note that around $61 \%$ of the domains in the random dataset are single page domains. Figure $5 \mathrm{~b}$ presents the cumulative fraction of URLs for the two datasets. Our results show that for the Alexa dataset, only 19000 domains cover $80 \%$ of all the URLs while, for the random dataset, only 10000 domains span the same percentage of URLs.

These results give strong empirical evidence (due to a large and random dataset) to previous results by Huberman and Adamic HA08, HA99 that demonstrate that the number of webpages per site is distributed according to a power law. For the random dataset, we fit a power-law distribution of the form:

$$
p(x)=\frac{\alpha-1}{x_{\min }}\left(\frac{x}{x_{\min }}\right)^{-\alpha},
$$

where, $x_{\min }=1$ and the parameter $\alpha$ is estimated as $\hat{\alpha}$ :

$$
\hat{\alpha}=1+n\left(\sum_{i=1}^{n} \ln \frac{x_{i}}{x_{\min }}\right)^{-1}=1.312
$$

where $\left\{x_{i}\right\}$ are the $n$ data points. The standard error of the estimate is given by:

$$
\sigma=\frac{\hat{\alpha}-1}{\sqrt{n}}=0.0004
$$

Figure $5 \mathrm{c}$ presents the number of unique decompositions per host for the two datasets. These numbers are very close to that of the number of URLs and hence verify a similar power law distribution. The domains that cover the majority of the decompositions contain URLs that are difficult to re-identify (due to Type I collisions). While, the domains representing the tail of the distribution provide URLs which can be re-identified with high certainty using only a few prefixes.

In Figure $5 \mathrm{~d}$, $5 \mathrm{e} 5 \mathrm{f}$, we present the mean, minimum and maximum number of decompositions of URLs per domain in the two datasets. We observe that $51 \%$ of the random domains present a maximum of 10 decompositions for a URL, while the same is true for $41 \%$ of the Alexa domains. The average number of decompositions for over $46 \%$ of the hosts from the two datasets lies in the interval $[1,5]$. Hence, a random URL on these hosts can generate on an average a maximum of $\left(\begin{array}{l}5 \\ 2\end{array}\right)=10$ Type I collisions on two prefixes. As a result, URLs on these hosts can be re-identified using only a few prefixes.

In Figure 6, we present the number of non-zero collisions on 32-bit digests among decompositions for the two datasets. We note that the probability of finding a collision is $\frac{1}{2^{16}}$ : due to birthday paradox. Hence, domains hosting more than $2^{16}$ decompositions generate collisions. In comparison to the Alexa dataset, the number of collisions is higher among the URLs of the random dataset for the first 1000 hosts. The tails of the two distribution curves are however close. We observe that only $0.48 \%$ of the domains of the Alexa dataset yield collisions, while the rate is $0.26 \%$ for the random dataset. This low collision rate among decompositions eventually helps in re-identification. 


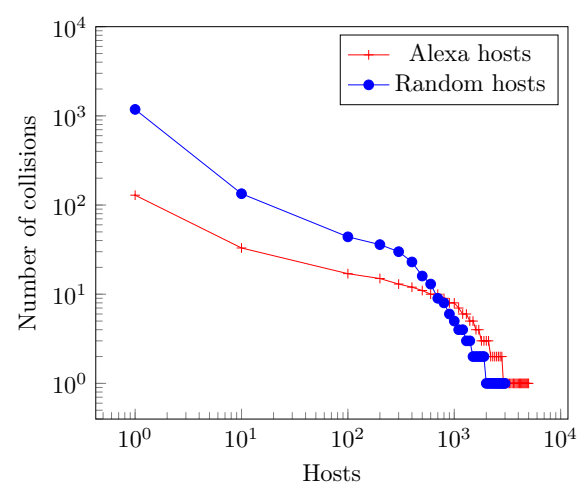

Figure 6: Non-zero collisions on digests' prefixes.

We now consider Type II collisions. A Type II collision occurs on URLs that share common decompositions. Thus, in order for a Type II collision to occur, the number of decompositions per domain must be at least $2^{32}$. However, the maximum number of decompositions per domain from either of the datasets is of the order of $10^{7}$ (see Figure 5c), which is smaller that $2^{32}$. This implies that Type II collisions do not occur for any of the hosts in our datasets.

As for Type I collisions, as expected, the number of collisions found was directly proportional to the number of unique decompositions on the host. On the one hand, we found several domains from both the datasets for which the number of such collisions was as high as 1 million, while on the other hand, we found over a hundred thousand domains for which the number of such collisions was less than 20. Hence, many of the non-leaf URLs on these hosts can be re-identified by inserting as less as 3 prefixes per URL. Interestingly, we observed that $56 \%$ of the domains in the random dataset do not have Type I collisions, while the same is true for around $60 \%$ of the domains from the Alexa dataset. Consequently, URLs on these domains can be easily re-identified using only 2 prefixes.

\subsection{A Tracking System based on SB}

Our analysis shows that it is possible to re-identify certain URLs whenever multiple prefixes corresponding to them are sent to the servers. Relying on this fact, GoogLE and YANDEX could potentially build a tracking system based on GSB and YSB. The robustness of the system would depend on the maximum number of prefixes per URL that they choose to include in the client's database. In the following, we denote this parameter by $\delta$. Clearly, larger is the $\delta$, more robust is the tracking tool. We note that its value is however bounded and chosen according to the memory constraints on the client's side.

The tracking system would essentially work as follows. First, Google and YANDEX choose the parameter $\delta \geq 2$, and build a shadow database of prefixes corresponding to at most $\delta$ decompositions of the targeted URLs. Second, they insert/push those prefixes in the client's database. Google and YAndex can identify individuals (using the SB cookie) each time their servers receive a query with at least two prefixes present in the shadow database.

The crucial point to address is how they may choose the prefixes for a given target URL. In Algorithm 1] we present a simple procedure to obtain these prefixes for a target URL given a bound $\delta$. The algorithm first identifies the domain that hosts the URL, which in most cases will be a Second-Level Domain (SLD) (see Line 2). Using their indexing capabilities, the SB providers then recover all URLs hosted on the domain and then obtain the set of unique decompositions 


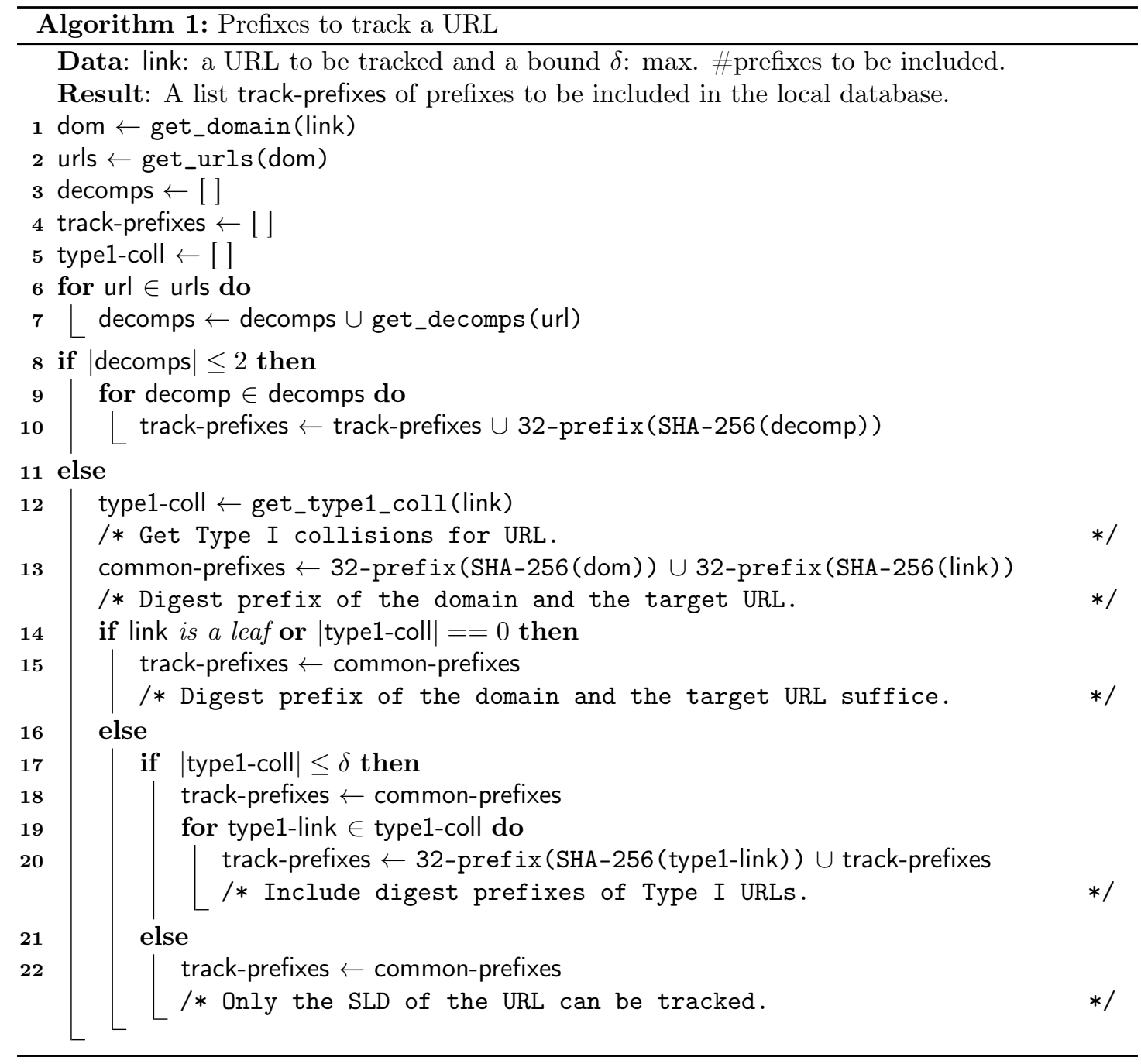

of the URLs (Line 3,6,7). Now, if the number of such decompositions is less than 3 , then the prefixes to be included for the target URL are those corresponding to these decompositions (Line 8-10). Otherwise, the algorithm determines the number of Type I collisions on the URL (Line 11-22). If no Type I collision exists or if the URL represents a leaf, then only two prefixes suffice to re-identify the URL. One of these prefixes is chosen to be the prefix of the URL itself and the other one can be that of any arbitrary decomposition. We however choose to include the prefix of the domain itself (Line 13-15). If the number of Type I collisions is non-zero but less than or equal to $\delta$, then the prefixes of these Type I URLs are also included (Line 17-20). Finally, if the number of Type I collisions is greater than $\delta$, then the URL cannot be precisely tracked. Nevertheless, including the prefix of the URL and its domain allows to re-identify the SLD with precision (Line 21-22). We note that if the prefixes are inserted according to this algorithm, the probability that the re-identification fails is $\left(\frac{1}{2^{32}}\right)^{\delta}$.

To illustrate the algorithm, let us use the URLs of PETS conference SLD. We first assume that the SB providers wish to identify participants who are interested in submitting a paper. Hence, 
the target URL is petsymposium.org/2016/cfp.php. The URL has 3 decompositions as given in Table 4. Since the target URL is a leaf, prefixes for the first and last decompositions would suffice to track a client visiting the target URL. Now, let us consider the case when the SB provider wishes to track a client's visit on petsymposium.org/2016 web page. At the time of writing the paper, the target URL yielded Type 1 collisions with: petsymposium.org/2016/links .php and petsymposium.org/2016/faqs.php (see Table 4). Thus, the SB provider would include the prefixes corresponding to these 2 URLs and that of petsymposium.org/2016 and the domain petsymposium.org/. In total only 4 prefixes suffice in this case. Now, whenever the SB server receives the last two prefixes, then it learns that the client has visited the target URL. Additionally, this allows the server to track the other 2 URLs that create Type I collisions.

In fact, it is also possible to re-identify a URL by aggregating the requests sent by the client. This can be achieved by exploiting the temporal correlation between the queries of a user. YANDEX and GOOGLE can identify the requests of a given user thanks to the SB cookie. A user visiting: petsymposium.org/2016/cfp.php (having prefix 0xe70ee6d1) is very likely to visit the submission website: petsymposium.org/2016/submission/(having prefix 0x716703db). Instead of looking at a single query, the SB server now needs to correlate two queries. A user making two queries for the prefixes $0 x e 70$ ee $6 \mathrm{~d} 1$ and $0 \times 716703 \mathrm{db}$ in a short period of time is planning to submit a paper.

\section{Blacklist Analysis}

In this section, we present an analysis of the blacklists provided by GoogLe and YANDEx. Our analysis has two specific objectives: recover the database of GOOGLE and YANDEX and identify URLs that match multiple prefixes in the local database.

\subsection{Inverting the Digests}

As a first step in our analysis, we recover the prefix lists of GoogLE and Yandex. We then use these lists to query their servers using their respective APIs. This allows us to obtain the lists of full digests.

Our second step is an attempt to identify the URLs which correspond to these prefixes. To this end, we harvested phishing and malware URLs, domains, and IP addresses from several sources and tested for their belonging to the blacklists of prefixes. The list of all our sources can be found in Cor. In total, we harvested 1240300 malware URLs, 151331 phishing URLs and 2488828 URLs of other categories from the BigBlackList big. Lastly, we obtained 106923807 SLDs from the DNS Census 2013 project dns13. The project provides a public dataset of registered domains and DNS records gathered in the years 2012-2013. We included the last dataset to determine the percentage of prefixes in the local database that correspond to SLDs. We reiterate that domain names and SLDs are uniquely re-identifiable. A description of all the datasets employed in our analysis is given in Table 9 .

Table 9: Dataset used for inverting 32-bit prefixes.

\begin{tabular}{l|r|r}
\hline Dataset & Description & \# entries \\
\hline \hline Malware list & malware & $1,240,300$ \\
\hline Phishing list & phishing & 151,331 \\
\hline BigBlackList & malw., phish., porno, others & $2,488,828$ \\
\hline DNS Census-13 & second-level domains & $106,923,807$ \\
\hline
\end{tabular}


The results of our experiments are shown in Table 10. The findings reveal that the reconstruction for GoOGLE prefixes using the Malware, Phishing and BigBlackList datasets is rather inconclusive: $5.9 \%$ for malwares and $0.1 \%$ for phishing websites. For YANDEX, the situation is better but the majority of the database still remains unknown. However, the DNS Census-13 dataset produces a much larger reconstruction for all the lists except that of the phishing database. The reconstruction is as high as $55 \%$ for YANDEX files. We highlight that phishing domains are often short-lived and since the DNS Census-13 dataset dates back to 2013, the result of the reconstruction for phishing lists is very limited, only $2.5 \%$ for GoOGLE and $5.6 \%$ for YANDEX. Nevertheless, these results demonstrate that 20\% of the GOOGLE malware list represents SLDs, while $31 \%$ of the prefixes in the YANDEX malware lists correspond to SLDs. Hence, these prefixes can be re-identified with very high certainty.

Table 10: Matches found with our datasets.

\begin{tabular}{|c|l|r|r|r|r|}
\cline { 3 - 5 } \multicolumn{1}{c|}{ list name } & \multicolumn{4}{c|}{ \#matches (\%match) } \\
\cline { 2 - 6 } \multicolumn{1}{c|}{} & \multicolumn{1}{|c|}{ Malware list } & Phishing list & BigBlackList & DNS Census-13 \\
\hline \multirow{5}{*}{ GOOGLE } & goog-malware-shavar & $18785(5.9)$ & $351(0.1)$ & $6208(1.9)$ & $63271(20)$ \\
\cline { 2 - 6 } & googpub-phish-shavar & $632(0.2)$ & $11155(3.5)$ & $816(0.26)$ & $7858(2.5)$ \\
\hline \multirow{5}{*}{ YANDEX } & ydx-malware-shavar & $44232(15.6)$ & $417(0.1)$ & $11288(3.9)$ & $88299(31)$ \\
\cline { 2 - 6 } & ydx-adult-shavar & $29(6.6)$ & $1(0.2)$ & $33(7.6)$ & $201(46.3)$ \\
\cline { 2 - 6 } & ydx-mobile-only-malware-shavar & $19(0.9)$ & $0(0)$ & $17(0.8)$ & $790(37.5)$ \\
\cline { 2 - 6 } & ydx-phish-shavar & $58(0.1)$ & $1568(4.9)$ & $153(0.47)$ & $1761(5.6)$ \\
\cline { 2 - 6 } & ydx-mitb-masks-shavar & $20(22.9)$ & $0(0)$ & $1(1.1)$ & $9(10.3)$ \\
\cline { 2 - 6 } & ydx-porno-hosts-top-shavar & $1682(1.6)$ & $220(0.2)$ & $11401(11.40)$ & $55775(55.7)$ \\
\cline { 2 - 6 } & ydx-sms-fraud-shavar & $66(0.6)$ & $1(0.01)$ & $22(0.20)$ & $1028(9.7)$ \\
\cline { 2 - 6 } & ydx-yellow-shavar & $43(20)$ & $1(0.4)$ & $8(3.8)$ & $76(36.4)$ \\
\hline
\end{tabular}

It is pertinent to compare the result of our reconstruction with a similar attempt with another list in the past. German censorship federal agency called BPjM maintains a secret list of about 3000 URLs believed to be unsuitable for women and children. The list is anonymized and distributed in the form of MD5 or SHA-1 hashes as the "BPJM-Modul" bpj]. Though similar to the lists handled by GOOGLE and YANDEX, hackers have been able retrieve $99 \%$ of the cleartext entries. We have applied the same approach, yet the reconstruction rate obtained has not been equally high. This proves that in order to reconstruct the database in cleartext, one would need high crawling capabilities and hence it is not realizable for general users. Furthermore, unlike the BPjM list, the blacklists provided by GSB and YSB are extremely dynamic. This requires a user to regularly crawl web pages on the web, which renders the reconstruction even more difficult.

\subsection{Orphan Prefixes}

We now look for orphan prefixes in YANDEX and Google Safe Browsing. We call an entry in the prefix list as orphan if no 256-bit digest matches it in the corresponding list of full digests. It is worth noticing that these prefixes cannot be termed as false positives. We also look for URLs in the Alexa list which generate an orphan prefix. Table 11 presents the results of our findings. Both Google and YANDEx have orphans. While GoOgle has 159 orphan prefixes, for YANDEX the numbers are astonishingly high. $43 \%$ for ydx-adult-shavar, $99 \%$ for ydx-phish-shavar, $95 \%$ for ydx-sms-fraud-shavar and $100 \%$ of the prefixes in ydx-mitb-masks-shavar and ydx-yellow-shavar are orphans. We did not find any URL in the Alexa list matching a Google orphan prefix. But there are $572+88$ URLs of the Alexa list with one parent: the prefix matches one full digest. For YANDEX, we found 271 URLs matching an orphan prefix and 20220 URLs with one parent. 
Table 11: Distribution of prefixes provided by Google and YANDEX as the number of full hashes per prefix. Collision with the Alexa list is also given.

\begin{tabular}{|c|c|c|c|c|c|c|c|c|c|}
\hline & \multirow[b]{2}{*}{ list name } & \multicolumn{3}{|c|}{ \#full hash per prefix } & & \multicolumn{3}{|c|}{ \#Coll. with Alexa list } & \multirow[b]{2}{*}{ Total } \\
\hline & & 0 & 1 & 2 & Total & 0 & 1 & 2 & \\
\hline \multirow{2}{*}{ GOOGLE } & goog-malware-shavar & 36 & 317759 & 12 & 317807 & 0 & 572 & 0 & 572 \\
\hline & googpub-phish-shavar & 123 & 312494 & 4 & 312621 & 0 & 88 & 0 & 88 \\
\hline \multirow{8}{*}{ YANDEX } & ydx-malware-shavar & 4184 & 279015 & 12 & 283211 & 73 & 2614 & 0 & 2687 \\
\hline & ydx-adult-shavar & 184 & 250 & 0 & 434 & 38 & 43 & 0 & 81 \\
\hline & ydx-mobile-only-malware-shavar & 130 & 1977 & 0 & 2107 & 2 & 22 & 0 & 24 \\
\hline & ydx-phish-shavar & 31325 & 268 & 0 & 31593 & 22 & 0 & 0 & 22 \\
\hline & ydx-mitb-masks-shavar & 87 & 0 & 0 & 87 & 2 & 0 & 0 & 2 \\
\hline & ydx-porno-hosts-top-shavar & 240 & 99750 & 0 & 99990 & 43 & 17541 & 0 & 17584 \\
\hline & ydx-sms-fraud-shavar & 10162 & 447 & 0 & 10609 & 76 & 3 & 0 & 79 \\
\hline & ydx-yellow-shavar & 209 & 0 & 0 & 209 & 15 & 0 & 0 & 15 \\
\hline
\end{tabular}

The presence of orphan prefixes is very difficult to justify. Moreover, the behavior of a browser on these prefixes is not consistent. Some of the orphan prefixes are considered as false positives by YANDEX while others are declared as true positives. There are three possible explanations to argue the presence of orphans. First, that there is an inconsistency between the prefix lists and the corresponding lists of full digests. This could be due to a misconfiguration, latency in the update or the result of a development error. This particularly might hold for GoogLE since very few prefixes are orphans. Second, that the services have intentionally noised the database in order to mislead attackers who may try to re-identify URLs from the prefixes. The last argument being that these SB providers might have tampered with their prefixes' database. The presence of large number orphans for YANDEX proves that it is possible to include any arbitrary prefix in the blacklists.

\subsection{Presence of Multiple Prefixes}

The inclusion of multiple prefixes for a URL is not a hypothetical situation. Instead, our experiments with the malicious databases show that Google and YANDEX indeed include multiple prefixes for a URL.

We employ the Alexa list of popular websites and the BigBlackList as test vectors for our experiments. The non-malicious Alexa list has been used in our experiments to determine if the GOOGLE or YANDEX indulge in any abusive use of SB.

In case of the Alexa list, we found 26 URLs on two domains, viz. 1001cartes.org and 1ptv.ru that create 2 hits each in the malware database of GoogLE. As for the phishing database, we found the URL: wps3b.17buddies.net that creates 2 hits. For YANDEX, we found 1352 such URLs distributed over 26 domains. 1158 of these URLs create hits in ydx-malware-shavar while the remaining 194 are hits in ydx-porno-hosts-top-shavar. We present a subset of these URLs in Table 12. The large number of such URLs is essentially due to Type I collisions. Nevertheless, these URLs are spread over several domains which shows that YANDEX actively includes several prefixes for a URL. This is however less evident for GoogLE. We reiterate that the corresponding domains and in some cases even the URLs are re-identifiable. This allows YANDEX, for instance, to learn whether a user prefers the adult site wickedpictures.com or mofos.com through domain re-identification. YANDEX can eventually determine the nationality of a person by the version of xhamster.com he visits. Finally, the most interesting example is that of teenslovehugecocks.com: the domain may allow YANDEX to identify pedophilic traits in a user. In case of BigBlackList, we found 103 URLs creating 2 hits in the YANDEX prefix lists. Moreover, we found one URL which creates 3 hits and another one which creates 4 hits. 
Table 12: A subset of URLs from the Alexa list with multiple matching prefixes in the GoogLE and YANDEX database.

\begin{tabular}{|c|c|c|c|}
\hline & URL & matching decomposition & prefix \\
\hline \multirow{6}{*}{ GOOGLE } & \multirow{2}{*}{ http://wps3b.17buddies.net/wp/cs_sub_7-2.pwf } & 17buddies.net/wp/cs_sub_7-2.pwf & $0 \times 18366658$ \\
\hline & & 17buddies.net/wp/ & $0 \times 77 \mathrm{c} 1098 \mathrm{~b}$ \\
\hline & \multirow{2}{*}{ http://www.1001cartes.org/tag/emergency-issues } & 1001cartes.org/tag/emergency-issues & $0 x a b 5140 c 7$ \\
\hline & & 1001cartes.org/tag/ & $0 \mathrm{xc} 73 \mathrm{e} 0 \mathrm{~d} 7 \mathrm{~b}$ \\
\hline & \multirow{2}{*}{ http://www.1ptv.ru/menu/ask/ } & www.1ptv.ru/ & $0 \times f 90449 \mathrm{~d} 7$ \\
\hline & & 1ptv.ru/menu/ & $0 \times \mathrm{xb} 15 \mathrm{dbc} 15$ \\
\hline \multirow{10}{*}{ YANDEX } & \multirow{2}{*}{ http://fr.xhamster.com/user/video } & fr.xhamster.com/ & 0xe4fdd86c \\
\hline & & xhamster.com/ & $0 \times 3074 \mathrm{e} 021$ \\
\hline & \multirow{2}{*}{ http://nl.xhamster.com/user/video } & nl.xhamster.com/ & 0xa95055ff \\
\hline & & xhamster.com/ & $0 \times 3074 \mathrm{e} 021$ \\
\hline & \multirow{2}{*}{ http://m.wickedpictures.com/user/login } & m.wickedpictures.com/ & $0 \times 7 e e 8 c 0 c c$ \\
\hline & & wickedpictures.com/ & 0xa7962038 \\
\hline & \multirow{2}{*}{ http://m.mofos.com/user/login } & m.mofos.com/ & $0 \times 6 e 961650$ \\
\hline & & mofos.com/ & $0 \times 00354501$ \\
\hline & \multirow{2}{*}{ http://mobile.teenslovehugecocks.com/user/join } & mobile.teenslovehugecocks.com/ & $0 \times 585667 a 5$ \\
\hline & & teenslovehugecocks.com/ & $0 \times 92824 b 5 c$ \\
\hline
\end{tabular}

\section{Related Work and Mitigations}

To the best of our knowledge no prior work has studied SB services from a privacy perspective. Due to the purpose and similarity of the service, our work is strongly related to web-search privacy. Indeed, the URLs visited by a user and the searched keywords reveal extensive information about users (see [JKPT07]). Several solutions used to improve web-search privacy can be applied to our case and most notably dummy requests (see $\mathrm{GSS}^{+} 14$ for an extended survey). This kind of solution is currently used by Firefox to query GSB. Each time Firefox makes a query to GSB, some dummy queries are also performed to hide the real one. The dummy requests are deterministically determined with respect to the real request to avoid differential analysis [Ved15]. This countermeasure can improve the level of $k$-anonymity for a single prefix match. However, re-identification is still possible in the case of multiple prefix match because the probability that two given prefixes are included in the same request as dummies is negligible.

Fixing GSB and YSB is not an easy task since private information retrieval is too costly $[\mathrm{SC07}$ Gol07, OG12]. To reduce the amount of information leak, we propose to query the server one-prefix-at-a-time. When a URL has several decompositions matching in the prefixes' database, the prefix corresponding to the root node/decomposition is first queried. Meanwhile, the targeted URL is pre-fetched by the browser and crawled to find if it contains Type I URLs. If the answer from GoOGLE or YANDEX is positive, a warning message is displayed to the user. Otherwise if Type I URLs exist, then the browser can query the server for the other prefixes. In this case, GoOGLE and YANDEx can only recover the domain but not the full URL. A message can be displayed to warn the users that the service may learn the URL he intends to visit.

\section{Conclusion}

Safe Browsing services are valuable tools to fight malwares, phishing and other online frauds. Unlike other Safe Browsing vendors, Google and YANDEx have made sincere efforts to render their services as private as possible. However, the effect of their anonymization efforts have been largely unclear. We have quantified the privacy provided by these services and have shown that the underlying anonymization technique of hashing and truncation fails when the server receives multiple prefixes for a URL.

Our observations on the YANDEx database and to a lesser extent on that of GoogLE show 
that it is possible to tamper these databases. These instances could either be deliberate attempts or the results of development errors/misconfigurations. Irrespective of the underlying cause, the service readily transforms into an invisible tracker that is embedded in several software solutions. As a future work, we want to design a plugin for Firefox and Chrome to make the users aware of the associated privacy issues.

\section{Acknowledgment}

This research was conducted with the support of LabEx PERSYVAL-Lab (ANR11-LABX-0025) and the project-team SCCyPhy.

\section{References}

[ale15] Alexa 1M Global Sites. Online, 2015. http://bit.1y/1yhXcgL.

[big] BigBlackList. Online. http://urlblacklist.com/.

[BLFM05] T. Berners-Lee, R. Fielding, and L. Masinter. Uniform Resource Identifier (URI): Generic Syntax. RFC 3986 (INTERNET STANDARD), January 2005. Updated by RFCs $6874,7320$.

[BLMM94] T. Berners-Lee, L. Masinter, and M. McCahill. Uniform Resource Locators (URL). RFC 1738, RFC Editor, December 1994. https://www.ietf.org/rfc/rfc1738.txt.

[Blo70] Burton H. Bloom. Space/Time Trade-offs in Hash Coding with Allowable Errors. Commun. ACM, 13(7):422-426, 1970.

[bpj] BPJM Modul. Online. http://bpjmleak.neocities.org/

[com15] Common Crawl. Online, 2015. http://commoncrawl.org/.

[Cor] Zeltser Security Corp. https://zeltser.com/malicious-ip-blocklists/.

[Cor13] Microsoft Corporation. Microsoft Security Intelligence Report. Technical report, Microsoft, December 2013. https://bit.1y/1qAfTgt.

[dns13] DNS Census 2013. Online, 2013. https://dnscensus2013.neocities.org/

[EO08] Gunes Ercal-Ozkaya. Routing in Random Ad-Hoc Networkds: Provably Better than Worst-case. PhD thesis, 2008.

[Gol07] I. Goldberg. Improving the Robustness of Private Information Retrieval. In Security and Privacy, 200\%. SEP '0\%. IEEE Symposium on, 2007.

[goo] Chromium bug. Online. https://code.google.com/p/chromium/issues/detail? id $=103243$.

[Goo12] Google. Google Safe Browsing Service in Mozilla Firefox Version 3. https:// developers.google.com/safe-browsing/firefox3_privacy?csw=1, April 2012.

[Goo14] Google. Google Chrome Privacy Notice. https://www.google.com/intl/en/ chrome/browser/privacy/, November 2014. 
[GSS $\left.{ }^{+} 14\right]$ Arthur Gervais, Reza Shokri, Adish Singla, Srdjan Capkun, and Vincent Lenders. Quantifying Web-Search Privacy. In ACM SIGSAC Conference on Computer and Communications Security, Scottsdale, AZ, USA, November 3-7, 2014, pages 966-977. ACM, 2014.

[HA99] Bernardo A. Huberman and Lada A. Adamic. Internet: Growth dynamics of the World-Wide Web. Nature, 401(6749):131-131, 1999.

[HA08] Bernardo A. Huberman and Lada A. Adamic. Internet: Growth dynamics of the World-Wide Web, 2008. http://arxiv.org/pdf/cond-mat/9901071v2.pdf.

[Inca] Facebook Inc. Link Shim. http://on.fb.me/1he2yEB.

[Incb] Google Inc. http://googleblog.blogspot.fr/2008/07/we-knew-web-was-big. html.

[Incc] Google Inc. Safe Browsing API. https://developers.google.com/ safe-browsing/

[Incd] Verisign Inc. http://www.verisigninc.com/en_US/innovation/dnib/index xhtml.

[Inc14] Google Inc. Google Transparency Report. Technical report, Google, June 2014. https://bit.1y/1A72tdQ.

[JKPT07] Rosie Jones, Ravi Kumar, Bo Pang, and Andrew Tomkins. "i know what you did last summer": query logs and user privacy. In ACM Conference on Information and Knowledge Management, CIKM 2007, Lisbon, Portugal, November 6-10, 2007, pages 909-914. ACM, 2007.

[McA] McAfee. McAfee Site Advisor. http://www.siteadvisor.com/.

$\left[\mathrm{MKD}^{+} 02\right]$ J. Mogul, B. Krishnamurthy, F. Douglis, A. Feldmann, Y. Goland, A. van Hoff, and D. Hellerstein. Delta encoding in HTTP. RFC 3229, RFC Editor, January 2002. http://tools.ietf.org/html/rfc3229

[moz] Mozilla bug. Online. https://bugzilla.mozilla.org/show_bug.cgi?id=368255

[Nat12] National institute of standards and technology. Secure Hash Standard (SHS). Technical Report FIPS PUB 180-4, National Institute of Standards \& Technology, march 2012.

[OG12] Femi Olumofin and Ian Goldberg. Revisiting the Computational Practicality of Private Information Retrieval. In Financial Cryptography and Data Security. Springer Berlin Heidelberg, 2012.

[Pro] Niels Provos. Safe Browsing - Protecting Web Users for 5 Years and Counting. http://googleonlinesecurity.blogspot.fr/2012/06/ safe-browsing-protecting-web-users-for.html

[RS98] Martin Raab and Angelika Steger. "Balls into Bins" - A Simple and Tight Analysis. In Proceedings of the Second International Workshop on Randomization and Approximation Techniques in Computer Science, RANDOM '98, pages 159-170, London,UK, 1998. Springer-Verlag. 
[SC07] Radu Sion and Bogdan Carbunar. On the Practicality of Private Information Retrieval. In Proceedings of the Network and Distributed System Security Symposium NDSS 200\%, San Diego, CA, USA, February 2007. The Internet Society.

[Swe02] Latanya Sweeney. k-Anonymity: A Model for Protecting Privacy. International Journal of Uncertainty, Fuzziness and Knowledge-Based Systems, 10(5):557-570, 2002.

[Sym] Symantec. Norton Safe Web. https://safeweb.norton.com/

[Ved15] Daniel Veditz. Personal Communication, 2015.

[WSa] Ltd WOT Services. Web of Trust. https://www.mywot.com.

[WSb] Ltd WOT Services. Web of Trust Privacy Policy. https://www.mywot.com/en/ privacy.

[Yan] Yandex. Yandex Safe Browsing. http://api.yandex.com/safebrowsing/ 


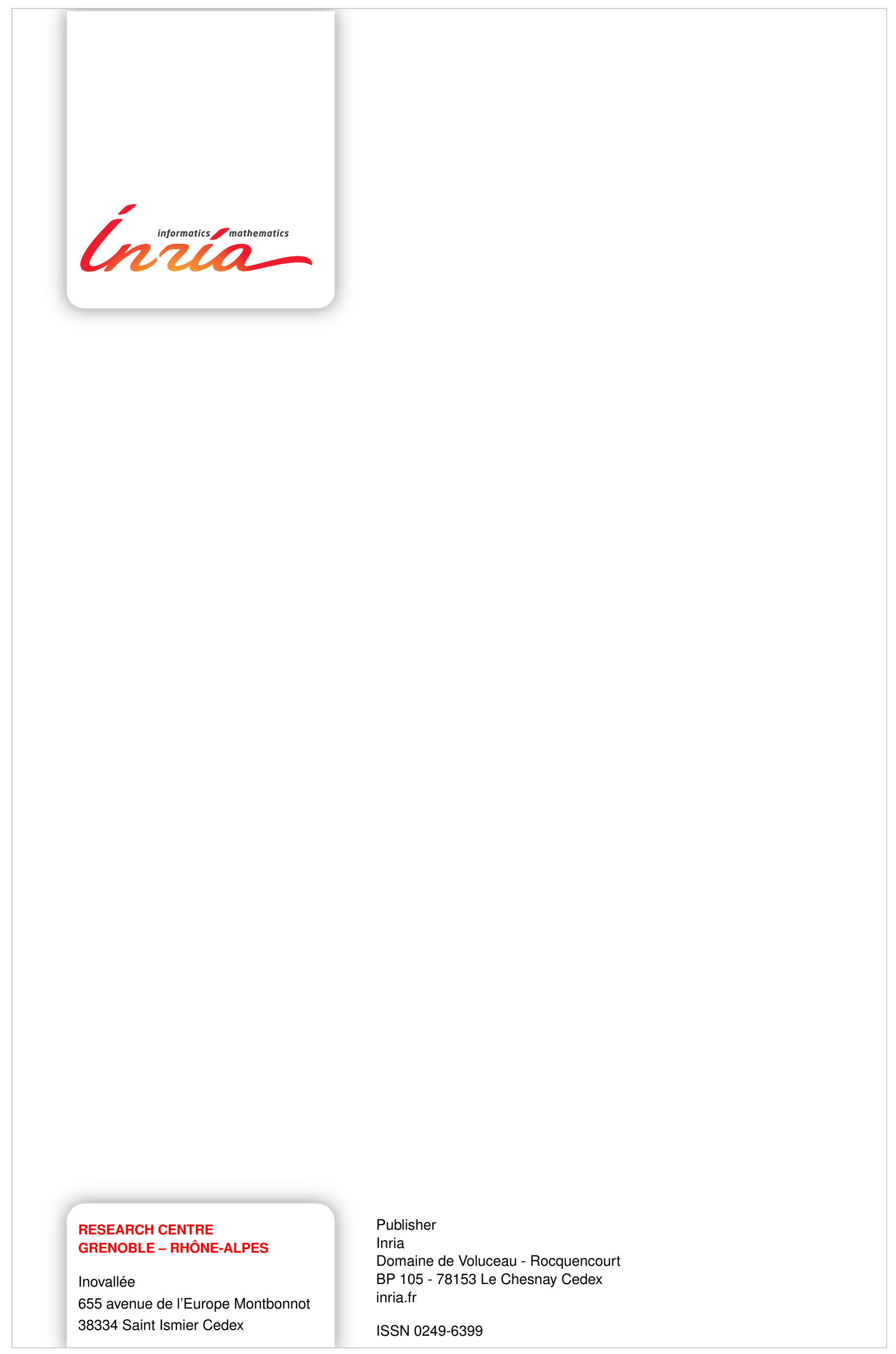

